\title{
Femtosecond two-dimensional infrared spectroscopy of synthetic hydrogen-bonded wires: From homogeneous to inhomogeneous dynamics
}

\author{
Stephan Knop, Martin Olschewski, and Peter Vöhringer \\ Institute for Physical and Theoretical Chemistry, University of Bonn, Wegelerstraße 12, 53115 \\ Bonn, Germany, p.voehringer@uni-bonn.de
}

\begin{abstract}
Femtosecond two-dimensional infrared (2DIR) spectroscopy was carried out on stereo-selectively synthesized poly-alcohols featuring a quasi-linear array of hydrogen-bonds. From the 2DIR spectra pump-frequency-dependent vibrational lifetimes are extracted, which in turn reflect the strength of the coupling between the hydroxyls constituting the H-bonded chain. The line-broadening dynamics reflect uniquely the conformational control of the scaffold supporting the H-bond wire and the resulting structural flexibility of the hydrogen-bond network.
\end{abstract}

\section{Introduction}

We recently introduced stereoselectively synthesized poly-alcohols as superb low-dimensional model systems for the structural dynamics and the vibrational spectroscopy of random hydrogenbond (H-bond) networks typically encountered in nature [1,2]. In these systems, a saturated hydrocarbon bearing a stereo-regular 2.4-anti-methylation pattern serves as a conformationally controlled scaffold to which a repetitive 1.3-diol substitution pattern is stereoselectively introduced. In the case where the hydroxyls are attached in an all-syn orientation, the simultaneous steric methyl repulsion and the dipolar attraction between adjacent $\mathrm{OHs}$ induced the spontaneous formation of an extended, almost linear H-bond network that remains structurally intact over extended time periods of tens of picoseconds. In contrast, diastereomers with an improper all-anti orientation of neighboring $\mathrm{OH}$ groups can also form an $\mathrm{H}$-bond network. This network is however, highly strained and features ultrafast dynamics of H-bond dissociation and reformation on time scales well below one picosecond. Here, we discuss the interrelation between vibrational line broadening dynamics and vibrational relaxation dynamics as exposed by femtosecond two-dimensional infrared spectroscopy (2DIR) in the hydroxyl stretching spectral region of the networks.

\section{Results and Discussion}

Firstly, we focus on the vibrational relaxation dynamics following an initial excitation of the $\mathrm{OH}$ stretching fundamental (see Fig. 1). In the all-syn case, the $\mathrm{OH}$-stretching vibrations and their transition dipoles are substantially coupled. As a result, before vibrational relaxation can fully set in, an $\mathrm{OH}$-excitation from an ultrafast resonant mid-IR pulse is rapidly redistributed among all the hydroxyl-groups constituting the H-bonded chain. This vibrational redistribution within the $\mathrm{OH}-$ 
stretching manifold is responsible for an ultrafast loss of memory regarding the frequency of initial excitation and as a result, an OH-vibrational lifetime is observed that depends only weakly on the frequency of the pump pulse. In essence, the measured lifetime represents always a thermal average over the set of four strongly coupled $\mathrm{OH}$-modes regardless of the frequency of excitation. In addition, anti-diagonal cuts through the bleaching region of the early-time 2DIR spectra of the allsyn polyol feature a rather large homogeneous linewidths (see Fig. 1, 0.4 ps). These are a direct consequence of the rather strong inter-hydroxylic couplings and a redistribution of energy among the set of $\mathrm{OH}$ oscillators that is rapid in comparison to the duration of the mid-IR pump pulse.

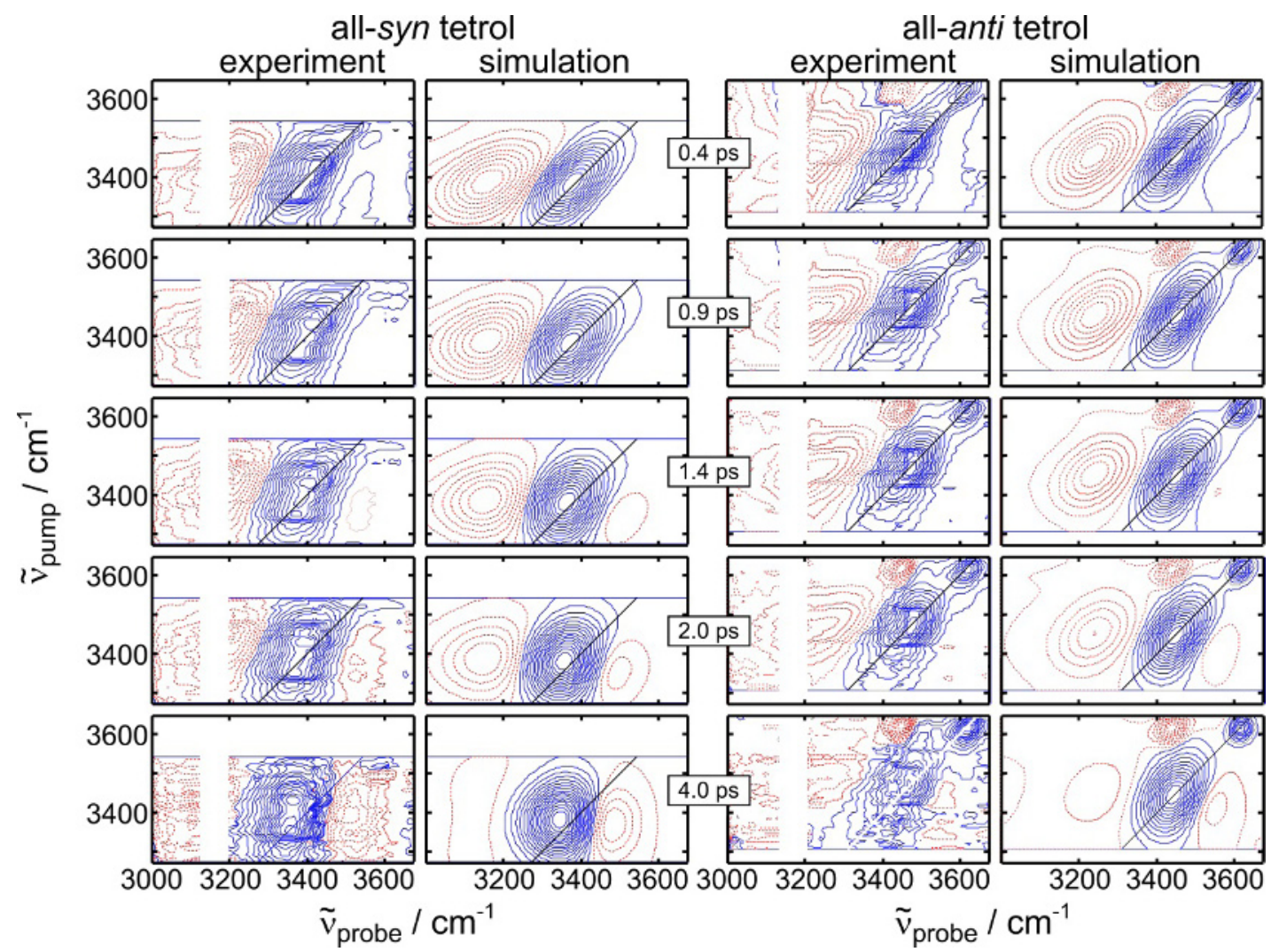

Fig. 1. Comparison between experimental and simulated 2DIR spectra of an all-syn tetrol (left pair of columns) and an all-anti tetrol (right pair of columns) for representative waiting delays.

Solid contours represent the bleaching and the stimulated emission of the $\mathrm{OH}$-stretching fundamental resonance while the dashed contours represent the anharmonically shifted induced absorption of the $\mathrm{OH}$-stretching excited state.

In contrast, in the all-anti diastereomers, the coupling of the OH-groups and their transition dipoles is much weaker. As a result, the $\mathrm{OH}$-excitation remains more or less localized on the initially excited $\mathrm{OH}$-oscillator for the time scale of the vibrational relaxation. A resonant narrowband mid-IR pulse is able to selectively excite from the inhomogeneously broadened (vide infra) resonance a specific sub-ensemble that relaxes with its own unique lifetime as defined by the sub-ensemble's instantaneous H-bond geometry. As a result inhomogeneous relaxation dynamics with a strongly pump-frequency-dependent lifetime are observed. In line with this interpretation, rather narrow holes with small homogeneous line widths are obtained from the anti-diagonal cuts through the 2DIR bleaching component of the all-anti polyol indicating that the rapid energy redistribution seen for the all-syn polyols can no longer occur because of the improper orientation of the neighboring $\mathrm{OH}$ groups. 
Interestingly, the 2DIR spectrum indicates that following the decay of the $\mathrm{v}=1$ excited state of the $\mathrm{OH}$ stretching mode, a pronounced induced absorption appears that is up-shifted in frequency relative to the ground-state bleach. This up-shifted absorption is an unambiguous fingerprint of the energy redistribution of the initial $\mathrm{OH}$-stretching quantum over the low-frequency modes of the poly-alcohol. A comparison of a thermal difference spectrum recorded at different temperatures with the 2DIR spectrum integrated over the pump frequency axis demonstrates that the molecules can be considered as "hot" after a few picoseconds. The appearance of the "blue"-shifted absorption has previously been attributed in alcohol oligomers [3] to the breakage of hydrogen bonds that is induced by the resonant pump-pulse. H-bond breakage may be facilitated via vibrational pre-dissociation [4], which couples the excited $\mathrm{OH}$-stretching excited state non-adiabatically to the C-C-O-H torsional continuum of the $\mathrm{OH}$ ground state. The $\mathrm{H}$-bond rupture generates a perturbed network whose $\mathrm{OH}-$ stretching resonance is shifted to higher frequencies because of the coupling of the OH-stretching transition dipole to the instantaneous H-bond geometries. Finally, a decay of the "hot" absorption corresponds to the structural relaxation of the disrupted network with reformation of all hydrogen bonds within the wire. By treating the relaxation of an $\mathrm{OH}$-stretching fundamental excitation as a non-adiabatic process that repopulates the vibrational ground state, the vibrational lifetime of hydrogen-bonded ethanol molecules in carbon tetrachloride solution could be determined mixed from quantum-classical molecular dynamics simulations within the framework of Fermi's golden rule [4]. The agreement between such kind of simulations and the experimental results is highly remarkable.

The heating contribution together with the decay of the $\mathrm{v}=1$ induced absorption gives rise to a highly characteristic time-dependent spectral shift of the ground-state hole, which serves as a critical benchmark for numerical simulations of the dynamically evolving 2DIR spectrum using the nonlinear response function formalism. Representative model simulations are also shown in Fig. 1. The agreement between experiment and simulation is satisfactory and is achieved using Gaussian line broadening dynamics only. However, an additional quasi-static inhomogeneous contribution is needed for simulating the 2DIR spectra of the all-anti diastereomers. The contribution is likely to result from an inhomogeneous distribution of hydrocarbon backbone conformations which essentially defines the strain within the H-bond wire. For the all-syn diastereomers, such an inhomogeneity is not found at all, which can be understood intuitively because only in the favorable all-syn configurations can the methyl repulsions and hydrogen-bonding interactions collectively to enforce a unique backbone conformation. Research involving molecular dynamics simulations of the dynamically evolving 2DIR line shape and its dependence on the temperature is currently ongoing.

\section{Acknowledgements}

Financial support by the Deutsche Forschungsgemeinschaft through the Collaborative Research Center SFB 624 "Templates: Functional Chemical Matrices" is gratefully acknowledged.

\section{References}

1. J. Seehusen, D. Schwarzer, J. Lindner, P. Vöhringer, Phys. Chem. Chem. Phys. 11, 8484 (2009)

2. S. Knop, T.L.C. Jansen, J. Lindner, P. Vöhringer, Phys. Chem. Chem. Phys. 13, 4641 (2011)

3. S. Woutersen, U. Emmerichs, H. J. Bakker, J. Chem. Phys. 107, 1483 (1997); .K. J. Gaffney, Paul H. Davis, I. R. Piletic, Nancy E. Levinger, M. D. Fayer J. Phys. Chem. A 106, 12012 (2002);

4. K. Kwac, E. Geva, J. Phys. Chem. B 116, 2856 (2012)

5. A. Staib, J.T. Hynes, Chem. Phys. Lett. 204, 197 (1993) 